\title{
Generalized Weak Transitivity of Preference
}

\author{
Thomas A. Runkler ${ }^{(凶)}$ \\ Siemens AG, Corporate Technology, Otto-Hahn-Ring 6, 81739 Munich, Germany \\ thomas.runkler@siemens.com
}

\begin{abstract}
Decision making processes are often based on (pairwise) preference relations. An important property of preference relations is transitivity. Many types of transitivity have been proposed in the literature, such as max-min and $\max -\max$ transitivity, restricted max-min and max-max transitivity, additive and multiplicative transitivity, or Łukasiewicz transitivity. This paper focuses on weak transitivity. Weak transitivity has been defined for additive preference relations. We extend this definition to multiplicative preference relations and further introduce a generalized version called generalized weak transitivity. We show that for reciprocal additive and multiplicative preference relations weak transitivity is equivalent to generalized weak transitivity, and we also illustrate generalized weak transitivity for preference relations that are neither additive nor multiplicative. Finally, we show how a total order (ranking of the options) can be constructed for any generalized weak transitive preference relation.
\end{abstract}

Keywords: Preference relations $\cdot$ Weak transitivity $\cdot$ Decision making

\section{Introduction}

Decision making processes are often based on preference relations $[2,6,7,9,14$, $17,18]$. Given a set of $n$ options, a (pairwise) preference relation is specified by an $n \times n$ preference matrix

$$
P=\left(\begin{array}{ccc}
p_{11} & \cdots & p_{1 n} \\
\vdots & \ddots & \vdots \\
p_{n 1} & \cdots & p_{n n}
\end{array}\right)
$$

where each matrix element $p_{i j} \geq 0$ quantifies the degree of preference of option $i$ over option $j$, where $i, j=1, \ldots, n$. We distinguish additive (or fuzzy) preference [21] and multiplicative preference [16]. An important property of preference relations is transitivity $[8,11]$. Given three options $i, j$, and $k$, transitivity specifies the relation between the preference of $i$ over $j, j$ over $k$, and $i$ over $k$. Many types of transitivity have been defined for preference relations, such as max-min and max-max transitivity [4,22], restricted $\max -\min$ and $\max -\max$ transitivity [18], additive and multiplicative transitivity [18], or Łukasiewicz transitivity [5, 13]. 
This paper focuses on weak transitivity [19]. We extend the definition of weak transitivity for additive preference relations to multiplicative preference relations (Definition 2). We derive an equivalent formulation of weak transitivity for reciprocal additive and multiplicative preference relations and use this to define a more general version of weak transitivity called generalized weak transitivity (Definition 3). We show that for reciprocal additive and multiplicative preference relations weak transitivity is equivalent to generalized weak transitivity (Theorems 1 and 2), and we also illustrate generalized weak transitivity for preference relations that are neither of additive nor multiplicative type. Finally, we show how a total order (ranking of the options) can be constructed for any generalized weak transitive preference relation (Theorem 3 ).

This paper is structured as follows: Sect. 2 briefly reviews additive preference relations and weak transitivity. Section 3 moves on to multiplicative preference relations and introduces a definition of weak transitivity for multiplicative preference relations. Section 4 develops a joint formula of weak transitivity for reciprocal additive and multiplicative preference relations and uses this to define generalized weak transitivity. Section 5 shows how a total order of elements can be constructed from any generalized weakly transitive preference relation. Finally, Sect. 6 summarizes our conclusions and outlines some directions for future research.

\section{Weakly Transitive Additive Preference}

Consider a preference matrix $P$. We call $P$ an additive preference matrix if and only if the following two conditions hold:

1. $p_{i j} \in[0,1]$ for all $i, j=1, \ldots, n$, and

2. $p_{i j}=0.5$ if and only if the options $i$ and $j$ are equivalent.

This implies that all elements on the main diagonal of $P$ are

$$
p_{i i}=0.5
$$

for all $i=1, \ldots, n$. We call 0.5 the neutral additive preference. An additive preference matrix $P$ is called reciprocal if and only if

$$
p_{i j}+p_{j i}=1
$$

for all $i, j=1, \ldots, n$. This always holds for $i=j$ because of (2), so it is sufficient to check this condition only for $i \neq j$. Several different types of transitivity have been defined for preference relations. In this paper we consider weak transitivity as defined by Tanino [19]:

Definition 1 (weakly transitive additive preference). An $n \times n$ additive preference matrix $P$ is called weakly transitive if and only if

$$
p_{i j} \geq 0.5 \wedge \quad p_{j k} \geq 0.5 \Rightarrow p_{i k} \geq 0.5
$$

for all $i, j, k=1, \ldots, n$, 
This can be interpreted as follows: if $i$ is preferred over $j$ and $j$ is preferred over $k$, then $i$ is preferred over $k$, where in all cases preference is not less than neutral. For reciprocal additive preference relations it is sufficient for weak transitivity to check (4) only for $i \neq j, j \neq k$, and $i \neq k$, because all other cases are trivial.

Consider for example the preference matrix

$$
P^{A}=\left(\begin{array}{lll}
0.5 & 0.7 & 0.9 \\
0.3 & 0.5 & 0.8 \\
0.1 & 0.2 & 0.5
\end{array}\right)
$$

All elements of $P^{A}$ are in the unit interval and all elements on the main diagonal are equal to the neutral additive preference 0.5 , so $P^{A}$ is an additive preference matrix. For the sums of preferences and reverse preferences we obtain

$$
\begin{aligned}
& p_{12}^{A}+p_{21}^{A}=0.7+0.3=1 \\
& p_{13}^{A}+p_{31}^{A}=0.9+0.1=1 \\
& p_{23}^{A}+p_{32}^{A}=0.8+0.2=1
\end{aligned}
$$

so $P^{A}$ is reciprocal. For the preferences not smaller than neutral we obtain

$$
p_{12}^{A} \geq 0.5, p_{23}^{A} \geq 0.5, p_{13}^{A} \geq 0.5
$$

so (4) holds and $P^{A}$ is weakly transitive. To summarize, $P^{A}$ is a weakly transitive reciprocal additive preference matrix.

Next consider the preference matrix

$$
P^{B}=\left(\begin{array}{lll}
0.5 & 0.2 & 0.7 \\
0.8 & 0.5 & 0.4 \\
0.3 & 0.6 & 0.5
\end{array}\right)
$$

All elements of $P^{B}$ are in the unit interval and all elements on the main diagonal are 0.5 , so $P^{B}$ is an additive preference matrix. The sums of preferences and reverse preferences are

$$
\begin{aligned}
& p_{12}^{B}+p_{21}^{B}=0.2+0.8=1 \\
& p_{13}^{B}+p_{31}^{B}=0.7+0.3=1 \\
& p_{23}^{B}+p_{32}^{B}=0.4+0.6=1
\end{aligned}
$$

so $P^{B}$ is reciprocal. However, for the preferences not smaller than neutral we obtain

$$
p_{13}^{B} \geq 0.5, p_{32}^{B} \geq 0.5, p_{12}^{B} \geq 0.5
$$

so (4) does not hold and $P^{B}$ is not weakly transitive. Hence, $P^{B}$ is a not weakly transitive reciprocal additive preference matrix. 


\section{Weakly Transitive Multiplicative Preference}

Consider again a preference matrix $P$. We call $P$ a multiplicative preference matrix if $p_{i j}=1$ if and only if the options $i$ and $j$ are equivalent. This implies that all elements on the main diagonal of $P$ are

$$
p_{i i}=1
$$

for all $i=1, \ldots, n$. We call 1 the neutral multiplicative preference. A multiplicative preference matrix $P$ is called reciprocal if and only if

$$
p_{i j} \cdot p_{j i}=1
$$

for all $i, j=1, \ldots, n$. This always holds for $i=j$ because of (15), so it is sufficient to check this condition only for $i \neq j$. We modify the condition (4) for weak transitivity of additive preference relations by replacing the neutral additive preference 0.5 by the neutral multiplicative preference 1 , and obtain

Definition 2 (weakly transitive multiplicative preference). An $n \times n$ multiplicative preference matrix $P$ is called weakly transitive if and only if

$$
p_{i j} \geq 1 \wedge \quad p_{j k} \geq 1 \Rightarrow \quad p_{i k} \geq 1
$$

for all $i, j, k=1, \ldots, n$.

For reciprocal multiplicative preference relations it is sufficient for weak transitivity to check (17) only for $i \neq j, j \neq k$, and $i \neq k$, because (just as for additive preference relations) all other cases are trivial.

Now consider the preference matrix

$$
P^{C}=\left(\begin{array}{ccc}
1 & 2 & 4 \\
1 / 2 & 1 & 3 \\
1 / 4 & 1 / 3 & 1
\end{array}\right)
$$

All elements on the main diagonal of $P^{C}$ are one, so $P^{C}$ is a multiplicative preference matrix. For the products of preferences and reverse preferences we obtain

$$
\begin{aligned}
& p_{12}^{C} \cdot p_{21}^{C}=2 \cdot 1 / 2=1 \\
& p_{13}^{C} \cdot p_{31}^{C}=4 \cdot 1 / 4=1 \\
& p_{23}^{C} \cdot p_{32}^{C}=3 \cdot 1 / 3=1
\end{aligned}
$$

so $P^{C}$ is reciprocal. For the preferences not smaller than neutral $(\geq 1$ for multiplicative preferences) we obtain

$$
p_{12}^{C} \geq 1, p_{23}^{C} \geq 1, p_{13}^{C} \geq 1
$$

so (17) holds and $P^{C}$ is weakly transitive. To summarize, $P^{C}$ is a weakly transitive reciprocal multiplicative preference matrix. 
As another example consider

$$
P^{D}=\left(\begin{array}{ccc}
1 & 1 / 3 & 4 \\
3 & 1 & 1 / 2 \\
1 / 4 & 2 & 1
\end{array}\right)
$$

which has ones on the main diagonal, so $P^{D}$ is a multiplicative preference matrix. The products of preferences and reverse preferences are

$$
\begin{aligned}
& p_{12}^{D} \cdot p_{21}^{D}=1 / 3 \cdot 3=1 \\
& p_{13}^{D} \cdot p_{31}^{D}=4 \cdot 1 / 4=1 \\
& p_{23}^{D} \cdot p_{32}^{D}=1 / 2 \cdot 2=1
\end{aligned}
$$

so $P^{D}$ is reciprocal. However, the preferences not smaller than neutral are

$$
p_{13}^{D} \geq 1, p_{32}^{D} \geq 1, p_{12}^{D} \geq 1
$$

so (17) is violated and $P^{D}$ is not weakly transitive. To summarize, $P^{D}$ is a not weakly transitive reciprocal multiplicative preference matrix.

\section{Generalized Weak Transitivity}

Let us revisit additive preference. An additive preference matrix $P$ is reciprocal if and only if (3)

$$
p_{i j}+p_{j i}=1
$$

for all $i, j=1, \ldots, n$, which implies

$$
p_{i j} \geq 0.5 \Rightarrow p_{j i}=1-p_{i j} \leq 0.5, \Rightarrow p_{i j} \geq p_{j i}
$$

and

$$
p_{i j} \geq p_{j i} \Rightarrow p_{i j} \geq 1-p_{i j} \Rightarrow 2 p_{i j} \geq 1 \Rightarrow p_{i j} \geq 0.5
$$

and so

$$
p_{i j} \geq 0.5 \Leftrightarrow p_{i j} \geq p_{j i}
$$

For reciprocal additive preference relations we can therefore rewrite the condition for weak transitivity (4)

$$
p_{i j} \geq 0.5 \wedge p_{j k} \geq 0.5 \Rightarrow p_{i k} \geq 0.5
$$

to the equivalent condition

$$
p_{i j} \geq p_{j i} \wedge p_{j k} \geq p_{k j} \Rightarrow p_{i k} \geq p_{k i}
$$

for all $i, j, k=1, \ldots, n$.

Now let us revisit multiplicative preference in the same way. A multiplicative preference matrix $P$ is reciprocal if and only if (16)

$$
p_{i j} \cdot p_{j i}=1
$$


for all $i, j=1, \ldots, n$, which implies

$$
p_{i j} \geq 1 \Rightarrow p_{j i}=1 / p_{i j} \leq 1 \Rightarrow p_{i j} \geq p_{j i}
$$

and

$$
p_{i j} \geq p_{j i} \Rightarrow p_{i j} \geq 1 / p_{i j} \Rightarrow p_{i j}^{2} \geq 1 \Rightarrow p_{i j} \geq 1
$$

and so

$$
p_{i j} \geq 1 \Leftrightarrow p_{i j} \geq p_{j i}
$$

For reciprocal multiplicative preference relations we can therefore rewrite the condition for weak transitivity (17)

$$
p_{i j} \geq 1 \wedge p_{j k} \geq 1 \Rightarrow p_{i k} \geq 1
$$

to the equivalent condition

$$
p_{i j} \geq p_{j i} \wedge p_{j k} \geq p_{k j} \Rightarrow p_{i k} \geq p_{k i}
$$

for all $i, j, k=1, \ldots, n$, which is equivalent to the condition (31) that we obtained for additive preference. So, for reciprocal additive preference and for reciprocal multiplicative preference we obtain the same condition $(31)=(35)$ for weak transitivity. This leads us to

Definition 3 (generalized weakly transitive preference). An $n \times n$ preference matrix $P$ is called generalized weakly transitive if and only if

$$
p_{i j} \geq p_{j i} \wedge p_{j k} \geq p_{k j} \Rightarrow p_{i k} \geq p_{k i}
$$

for all $i, j, k=1, \ldots, n, i \neq j, j \neq k, i \neq k$.

Notice that we exclude the cases $i=j, j=k$, and $i=k$ here, because these are trivial for reciprocal additive and multiplicative preference relations, and so we also want to exclude them for arbitrary preference relations. This means that elements on the main diagonal of $P$ are irrelevant for generalized weak transitivity.

It is easy to check that $P_{A}(5)$ and $P_{C}$ (18) satisfy generalized weak transitivity, and $P_{B}$ (10) and $P_{D}(23)$ do not. In general, the following two theorems relate weakly transitive additive and multiplicative preference to generalized weakly transitive preference.

Theorem 1 (additive generalized weak transitivity). A reciprocal additive preference matrix is weakly transitive if and only if it is generalized weakly transitive.

Theorem 2 (multiplicative generalized weak transitivity). A reciprocal multiplicative preference matrix is weakly transitive if and only if it is generalized weakly transitive.

Proof. The proof for both Theorems follows immediately from the equivalence of Eqs. (31), (35), and (36). 
So, for reciprocal additive and multiplicative preference matrices weak transitivity and generalized weak transitivity are equivalent. Therefore, let us now look at preference matrices which are neither additive nor multiplicative. Consider for example the preference matrix

$$
P^{E}=\left(\begin{array}{lll}
1 & 6 & 9 \\
2 & 4 & 8 \\
5 & 3 & 7
\end{array}\right)
$$

The elements on the main diagonal of $P^{E}$ are different from 0.5 and 1 , so $P^{E}$ is neither an additive preference matrix nor a multiplicative preference matrix. However, it may seem reasonable to interpret $P^{E}$ as a preference matrix. Imagine for example that the rows and columns of this matrix correspond to soccer teams, and each element $p_{i j}^{E}$ corresponds to the number of times that team $i$ has won over team $j$. The elements on the main diagonal are chosen arbitrarily and may be ignored for generalized weak transitivity, as pointed out above. For the offdiagonal preference pairs we have

$$
p_{12}^{E} \geq p_{21}^{E}, p_{23}^{E} \geq p_{32}^{E}, p_{13}^{E} \geq p_{31}^{E}
$$

so (36) holds and therefore $P^{E}$ is generalized weakly transitive.

As another example consider

$$
P^{F}=\left(\begin{array}{lll}
1 & 5 & 8 \\
9 & 4 & 2 \\
3 & 6 & 7
\end{array}\right)
$$

Again, the main diagonal is different from 0.5 and 1 , so this is neither an additive preference matrix nor a multiplicative preference matrix. For the off-diagonal preference pairs of $P_{F}$ we obtain

$$
p_{13}^{F} \geq p_{31}^{F}, p_{32}^{F} \geq p_{23}^{F}, p_{12}^{F} \geq p_{21}^{F}
$$

so $(36)$ is violated and therefore $P^{F}$ is not generalized weakly transitive.

\section{Total Order Induced by Generalized Weak Transitivity}

Generalized weak transitivity is an important property of preference relations because it allows to construct a total order (ranking) of the elements.

Theorem 3 (total order for generalized weak transitivity). If an $n \times n$ preference matrix is generalized weakly transitive, then we can construct a total order $o$ of the $n$ elements so that $o_{1} \geq o_{2} \geq \ldots \geq o_{n}$.

Proof. If a preference matrix $P$ is generalized weakly transitive, then from (36) follows that for each $i, j, k=1, \ldots, n$ with $i \neq j, j \neq k, i \neq k$. we will have at least one of the following six cases: 


$$
\begin{gathered}
p_{i j} \geq p_{j i}, p_{j k} \geq p_{k j}, p_{i k} \geq p_{k i} \Rightarrow o_{i} \geq o_{j} \geq o_{k} \\
p_{i k} \geq p_{k i}, p_{k j} \geq p_{j k} p_{i j} \geq p_{j i} \Rightarrow o_{i} \geq o_{k} \geq o_{j} \\
p_{j i} \geq p_{i j}, p_{i k} \geq p_{k i}, p_{j k} \geq p_{k j} \Rightarrow o_{j} \geq o_{i} \geq o_{k} \\
p_{j k} \geq p_{k j}, p_{k i} \geq p_{i i}, p_{j i} \geq p_{i j} \Rightarrow o_{j} \geq o_{k} \geq o_{i} \\
p_{k i} \geq p_{i k}, p_{i j} \geq p_{j i}, p_{k j} \geq p_{j k} \Rightarrow o_{k} \geq o_{i} \geq o_{j} \\
p_{k j} \geq p_{j k}, p_{j i} \geq p_{i j}, p_{k i} \geq p_{i k} \Rightarrow o_{k} \geq o_{j} \geq o_{i}
\end{gathered}
$$

If more than one of these cases is satisfied, then we have ties $p_{i j}=p_{j i}$ or $p_{j k}=p_{k j}$ or $p_{i k}=p_{k i}$, which implies $o_{i}=o_{j}$ or $o_{j}=o_{k}$ or $o_{i}=o_{k}$, respectively, so the order may not be strict. For an arbitrary generalized weakly transitive preference matrix we can therefore pick arbitrary three elements $i, j, k$ and construct a total order for these. If we have a total order for $p \geq 3$ elements $i_{1}, i_{2}, \ldots, i_{p}$, then for any additional element $k$ from (36) follows that we will have one at least of the following three cases

$$
\begin{aligned}
& p_{k i_{1}} \geq p_{i_{1} k}, p_{i_{1} i_{2}} \geq p_{i_{2} i_{1}}, p_{k i_{2}} \geq p_{i_{2} k} \Rightarrow o_{k} \geq o_{i_{1}} \geq o_{i_{2}} \\
& p_{i_{p-1} i_{p}} \geq p_{i_{p} i_{p-1}}, p_{i_{p} k} \geq p_{k i_{p}}, p_{i_{p-1} k} \geq p_{k i_{p-1}} \Rightarrow o_{i_{p-1}} \geq o_{i_{p}} \geq o_{k}
\end{aligned}
$$

or we can find an index $j \in\{1,2, \ldots, p-1\}$ for which

$$
p_{i_{j} k} \geq p_{k i_{j}}, p_{k i_{j+1}} \geq p_{i_{j+1} k}, p_{i_{j} i_{j+1}} \geq p_{i_{j+1} i_{j}} \Rightarrow o_{i_{j}} \geq o_{k} \geq o_{i_{j+1}}
$$

In the first case, we can insert element $k$ before element $i_{1}$ and obtain the new total order $k, i_{1}, i_{2}, \ldots, i_{p}$. In the second case, we can insert element $k$ after element $i_{p}$ and obtain the new total order $i_{1}, i_{2}, \ldots, i_{p}, k$. And in the third case we can insert element $k$ between elements $i_{j}$ and $i_{j+1}$ and obtain the new total order $i_{1} \ldots, i_{j}, k, i_{j+1} \ldots, i_{p}$. Again, several of these three cases may be satisfied if we have ties $p_{k i_{1}}=p_{i_{1} k}$ or $p_{k i_{p-1}}=p_{i_{p-1} k}$ or $p_{k i_{j}}=p_{i_{j} k}$ or $p_{k i_{j+1}}=p_{i_{j+1} k}$, which lead to equal ranks $o_{k}=o_{i_{1}}$ or $o_{k}=o_{i_{p-1}}$ or $o_{k}=o_{i_{j}}$ or $o_{k}=o_{i_{j+1}}$, respectively, so the order may be not strict. Using this scheme we can construct a total order for any generalized weakly transitive preference matrix.

For example, the preference matrix $P^{E}(37)$ is generalized weakly transitive, so we have the relation (38) for the off-diagonal preference pairs which yields the total order $o_{1} \geq o_{2} \geq o_{3}$. In the semantic context of the application we can interpret this as a ranking of soccer teams, where team 1 is ranked on top, then team 2 , and finally team 3 . Notice again that the resulting total order may be not strict, so the ranking can contain ties. As another example, the preference matrix $P^{F}$ (39) is not generalized weakly transitive, so the relation (40) for the off-diagonal preference pairs does not yield a total order, since $o_{1} \geq o_{3} \geq o_{2}$ contradicts $o_{1} \nsupseteq o_{2}$. Here, it is not possible to construct a ranking of the soccer teams. 


\section{Conclusions}

We have extended the concept of weak transitivity from additive preference relations to multiplicative preference relations and further in a generalized version to arbitrary preference relations. We have shown that generalized weak transitivity is an important property of preference relations because it allows to construct a strict order of elements (ranking of options) which is very useful in decision making processes.

Many questions have been left open for future research, such as:

- What are efficient algorithms to test a preference matrix for generalized weak transitivity and to construct the corresponding total order?

- How does generalized weak transitivity relate to other mathematical properties of preference relations such as monotonicity or positivity?

- How can weakly transitive preference relations be constructed from utilities [10] or from rank orders [12]?

- How do these types of transitivity extend to interval valued preferences $[1,3$, $15,20]$ ?

\section{References}

1. Bilgiç, T.: Interval-valued preference structures. Eur. J. Oper. Res. 105(1), 162183 (1998)

2. Brans, J.-P., Vincke, P., Mareschal, B.: How to select and how to rank projects: the PROMETHEE method. Eur. J. Oper. Res. 24(2), 228-238 (1986)

3. Cavallo, B., Brunelli, M.: A general unified framework for interval pairwise comparison matrices. Int. J. Approximate Reasoning 93, 178-198 (2018)

4. Dubois, D., Prade, H.: Fuzzy Sets and Systems. Academic Press, London (1980)

5. Duddy, C., Piggins, A.: On some oligarchy results when social preference is fuzzy. Soc. Choice Welf. 51(4), 717-735 (2018). https://doi.org/10.1007/s00355-0181134-4

6. Fürnkranz, J., Hüllermeier, E.: Preference learning. In: Sammut, C., Webb, G.I. (eds.) Encyclopedia of Machine Learning, pp. 789-795. Springer, Boston (2010). https://doi.org/10.1007/978-0-387-30164-8_662

7. Herrera, F., Herrera-Viedma, E., Chiclana, F.: Multiperson decision-making based on multiplicative preference relations. Eur. J. Oper. Res. 129(2), 372-385 (2001)

8. Herrera-Viedma, E., Herrera, F., Chiclana, F., Luque, M.: Some issues on consistency of fuzzy preference relations. Eur. J. Oper. Res. 154(1), 98-109 (2004)

9. Orlovsky, S.A.: Decision-making with a fuzzy preference relation. Fuzzy Sets Syst. 1(3), 155-167 (1978)

10. Runkler, T.A.: Constructing preference relations from utilities and vice versa. In: Carvalho, J.P., Lesot, M.-J., Kaymak, U., Vieira, S., Bouchon-Meunier, B., Yager, R.R. (eds.) IPMU 2016. CCIS, vol. 611, pp. 547-558. Springer, Cham (2016). https://doi.org/10.1007/978-3-319-40581-0_44

11. Runkler, T.A.: Mapping utilities to transitive preferences. In: Medina, J., et al. (eds.) IPMU 2018. CCIS, vol. 853, pp. 127-139. Springer, Cham (2018). https:// doi.org/10.1007/978-3-319-91473-2_11 
12. Runkler, T.A.: Canonical fuzzy preference relations. In: Kearfott, R.B., Batyrshin, I., Reformat, M., Ceberio, M., Kreinovich, V. (eds.) IFSA/NAFIPS 20192019. AISC, vol. 1000, pp. 542-555. Springer, Cham (2019). https://doi.org/10.1007/ 978-3-030-21920-8_48

13. Runkler, T.A.: Generating preference relation matrices from utility vectors using Łukasiewicz transitivity. In: Kóczy, L.T., Medina-Moreno, J., Ramírez-Poussa, E., Šostak, A. (eds.) Computational Intelligence and Mathematics for Tackling Complex Problems. SCI, vol. 819, pp. 123-130. Springer, Cham (2020). https://doi. org/10.1007/978-3-030-16024-1_16

14. Runkler, T.A., Chen, C., Coupland, S., John, R.: Just-in-time supply chain management using interval type-2 fuzzy decision making. In: IEEE International Conference on Fuzzy Systems, New Orleans, Louisiana, USA, pp. 1149-1154, June 2019

15. Runkler, T.A., Coupland, S., John, R.: Interval type-2 fuzzy decision making. Int. J. Approximate Reasoning 80, 217-224 (2017)

16. Saaty, T.L.: Analytic hierarchy process. In: Gass, S.I., Fu, M.C. (eds.) Encyclopedia of Operations Research and Management Science, pp. 52-64. Springer, Boston (2013). https://doi.org/10.1007/978-1-4419-1153-7_31

17. Sousa, J.M., Palm, R.H., Silva, C.A., Runkler, T.A.: Optimizing logistic processes using a fuzzy decision making approach. IEEE Trans. Syst. Man Cybern. A 33(2), 245-256 (2003)

18. Tanino, T.: Fuzzy preference orderings in group decision making. Fuzzy Sets Syst. 12(2), 117-131 (1984)

19. Tanino, T.: Fuzzy preference relations in group decision making. In: Kacprzyk, J., Roubens, M. (eds.) Non-Conventional Preference Relations in Decision Making. Lecture Notes in Economics and Mathematical Systems, vol. 301, pp. 54-71. Springer, Heidelberg (1988). https://doi.org/10.1007/978-3-642-51711-2_4

20. Türkşen, İ.B., Bilgiç, T.: Interval valued strict preference with Zadeh triples. Fuzzy Sets Syst. 78(2), 183-195 (1996)

21. Zadeh, L.A.: Similarity relations and fuzzy orderings. Inf. Sci. 3(2), 177-200 (1971)

22. Zimmermann, H.J.: Fuzzy Set Theory and Its Applications. Kluwer Academic Publishers, Boston (1985) 